\title{
Evaluation of Changes in Refractive Errors Before and After Trabeculectomy and Shunt Surgery
}

This article was published in the following Dove Press journal: Clinical Optometry

\author{
Farzaneh Dehghanian \\ Nasrabadi ${ }^{\prime}$ \\ Hassan Hashemi ${ }^{\text {I }}$ \\ Mohammad Aghazadeh \\ Amiri (iD ${ }^{2}$ \\ Ghasem Fakhraie ${ }^{3}$ \\ Alireza Akbarzadeh \\ Baghban (iD ${ }^{4}$ \\ Ebrahim Jafarzadehpour ${ }^{\prime}$ \\ Abbasali Yekta ${ }^{5}$ \\ Mehdi Khabazkhoob (1D ${ }^{6}$ \\ 'Noor Research Center for Ophthalmic \\ Epidemiology, Noor Eye Hospital, Tehran, \\ Iran; ${ }^{2}$ Department of Optometry, Faculty \\ of Rehabilitation Science, Shahid Beheshti \\ University of Medical Science, Tehran, \\ Iran; ${ }^{3}$ Farabi Eye Hospital, Tehran \\ University of Medical Science, Tehran, \\ Iran; ${ }^{4}$ Proteomics Research Center, \\ School of Rehabilitation, Shahid Beheshti \\ University of Medical Science, Tehran, \\ Iran; ${ }^{5}$ Department of Optometry, \\ Mashhad University of Medical Science, \\ Mashhad, Iran; ${ }^{6}$ Department of Medical \\ Surgical Nursing, School of Nursing and \\ Midwifery, Shahid Beheshti University of \\ Medical Sciences, Tehran, Iran
}

Correspondence: Mehdi Khabazkhoob Department of Medical Surgical Nursing, School of Nursing and Midwifery, Shahid Beheshti University of Medical Sciences, Tehran, Iran

Email khabazkhoob@yahoo.com

\begin{abstract}
Purpose: The aim of the present study was to investigate the change rate of astigmatism and axial, myopia, and hypermetropia in trabeculectomy and shunt surgery.

Patients and Methods: In this comparative study, two groups of 16 patients with an average age of $52.4 \pm 8.9$ and $94.6 \pm 6.7$ respectively underwent trabeculectomy and shunt surgery. The changes of reactive error were compared before the surgery and 1 week, 1 month, and 3 months after the surgery. Data from the two groups were analyzed via Friedman and Mann-Whitney tests.

Results: Average of intraocular pressure (IOP) before, and 1 week, 1 month, and 3 months after trabeculectomy is $31.31 \pm 7.6,9.8 \pm 2.8,10.5 \pm 1.29,10.9 \pm 1.26(P<0.05)$ and shunt is $36.06 \pm 10.32,13.5 \pm 3.3,11.68 \pm 2.21,11.18 \pm 1.27(P<0.05)$. Average astigmatism in trabeculectomy is $-1.00 \pm 0.59,-6.00 \pm 1.9,-2.21 \pm 1.18,-1.37 \pm 0.62(P<0.05)$ and in shunt it is -0.89 $\pm 0.46,-1.56 \pm 1.62,-1.51 \pm 1.46,-1.07 \pm 0.85(P>0.05)$. Percent WRT astigmatism in trabeculectomy is $37.5,100,37.5,31.25$ and in shunt it is $43.75,50,50,56.25$ and average of spherical equivalence (SE) in trabeculectomy is $-0.31 \pm 1.49,1.43 \pm 1.94,0.27 \pm 1.74,0.04$ $\pm 1.46(P<0.05)$ and in shunt it is $0.65 \pm 1.5,1.03 \pm 2.03,-0.70 \pm 1.64,0.62 \pm 1.54(P<0.05)$. Astigmatism and axial comparisons between trabeculectomy and shunt with Mann-Whitney test were significant $(P<0.05)$, but SE was not significant $(P>0.05)$.

Conclusion: The surgeries induced hypermetropia and reduction with the passing of time but astigmatism amount and percent of with-the-rule astigmatism in trabeculectomy were more than in shunt surgery.
\end{abstract}

Keywords: astigmatism, trabeculectomy, shunt surgery

\section{Introduction}

Glaucoma denotes a group of diseases that have the characteristic of optic neuropathy with associated visual function loss. ${ }^{1}$ The prevalence of glaucoma was $3.54 \%$ and until 2020, it has affected about 76 million persons. ${ }^{2,3}$ One of the primary risk factors of glaucoma is the elevation of intraocular pressure (IOP). Generally, increased IOP is caused by resistance to aqueous humor outflow. ${ }^{1}$

Glaucoma is classified into primary, secondary, and congenital categories. Primary glaucoma, unlike secondary glaucoma, is not associated with ocular and systemic disorders. ${ }^{1}$

The two procedures for the treatment of these diseases are trabeculectomy and Ahmed valve surgery. ${ }^{4}$

Trabeculectomy has become the gold standard surgical procedure since $1968 .{ }^{5}$ It is a filtering procedure to lower the IOP by making a new channel between the anterior chamber and subtenant space for the outflow of the aqueous humor. ${ }^{6}$ 
In 1973, Molten introduced the concept of draining the fluid away. Today, all available glaucoma drainage devices are based on the concept introduced by Molten. ${ }^{7}$ Glaucoma drainage devices (GDD) with a valve are designed to reduce postoperative hypotonic. Ahmed valve implants were introduced in 1993. In this procedure, the plate of the implant is in the sub-Tenon space and tube insertion is done in the anterior chamber for the outflow of the aqueous humor. Because of changes in refraction, astigmatism especially influences vision. So, various studies have investigated the refraction of eye after surgery of glaucoma. ${ }^{8,10}$

Kumari reported that trabeculectomy induced with-therule astigmatism, and many studies showed that the anterior-posterior length of the eye decreased after this surgery. ${ }^{11}$

Cunliffe said that trabeculectomy induced myopia and the amount of myopia and eye length are inversely related. ${ }^{12}$ Meanwhile, Popa said that trabeculectomy does not change the refractive error. ${ }^{13}$ Mokhtari investigated topographic corneal images before and after shunt surgery, which showed no change in corneal astigmatism. ${ }^{14}$

Studies about spherical refractive error after trabeculectomy have contradictory results and have not reported on the spherical refractive error after shunt. No study has ever aimed to compare changes in refractive error between shunt and trabeculectomy surgeries. However, having good visual acuity and no postoperative refractive error changes will have a significant impact on patient performance. And it is important to find an effective surgical procedure that, in addition to lowering the IOP on the eye, causes the least changes in the refractive power of the eye.

So this study investigates a comparison of changes in refractive error, corneal keratometry, and astigmatism before and 1 week, 1 month, and 3 months after trabeculectomy and shunt surgeries.

\section{Patients and Methods}

This research was an analytical cross-sectional study that was done in a time span between September 2015 to May 2016. Patients were selected from the clinic of glaucoma in Farabi Eye Hospital in Tehran, Iran. The purpose of the study was explained to the patients and those who agreed to participate signed an informed consent form.

A total of 32 eyes with both trabeculectomy and shunt ( $\mathrm{n}$ trabeculectomy $=16, \mathrm{n}$ shunt $=16$ ) were included in the study. Exclusive criteria were previous history of ocular trauma, intraocular surgery, and pathologic ocular disorder. Inclusive criteria were range of age $30-80$ years, visual acuity better than $4 / 10$, and high IOP or signs of glaucoma in the retina that may require trabeculectomy or shunt surgery. There were no patients who underwent cataract surgery simultaneously. There was no gender specification. Before the surgery, IOP was measured three times by Goldman application (Topcon slit lamp) and central corneal K-reading was measured by an autorefractor keratometer (Topcon, KR-800) and Javal keratometer.

Refraction was done three times by retinoscopy (Heine, Beta200) and auto refractor meter (Topcon, KR-800), and the mean was reported as the result. In anterior segment examination by slit lamp (Topcon), cornea, lens, anterior chamber depth, and angle were evaluated by an ophthalmologist. In gonioscopy the anterior angle was graded by Schaeffer's criteria. Forty minutes before posterior segment evaluation, one drop midrax $1 \%$ was instilled in the eye. Next, optic nerve head, cup to disc ratio, vessels of retina, and macula were evaluated. Visual acuity was measured liner with and without correction (monocular) by the Snellen chart at 6-m distance.

All measurements were done before surgery, and 1 week, 1 month, and 3 months after shunt and trabeculectomy surgery.

The fornix-based trabeculectomy procedure was selected. General anesthesia was used as normal. A $6 \mathrm{~mm}$ limbalbased conjunctival flap was turned down on the cornea and the limbus was cleaned of a Tenon capsule. A $5 \times 3 \mathrm{~mm}$ partial-thickness rectangular scleral flap was dissected. The bed of this trapdoor now consists of the deep sclera-lamella and $4 \times 2 \mathrm{~mm}$ trabeculectomy was performed. The sides of this rectangle were carefully deepened, preferably under microscope control. Then, the rectangle of tissue is seized with fine-toothed forceps and removed by elevation away from the anterior chamber. A peripheral iridectomy was then performed. The scleral flap was secured and then conjunctiva was sutured by $8 / 0$ silk nylon. In shunt surgery, in the super temporal quadrant, a fornix-based conjunctival flap and Tenon were dissected. The tube was primed using a balanced salt solution. The plate was secured to the sclera with its anterior edge $6 \mathrm{~mm}$ posterior to the limbus using 7.0 Prolene sutures. A sclera tunnel was created starting approximately $2 \mathrm{~mm}$ from the limbus and, using a 23-gauge or 25-gauge needle the tunnel was advanced into the anterior chamber of the eye. The tube was trimmed bevel up and inserted through the tunnel into the anterior chamber aiming towards the center of the pupil. The tube was not ligated. A donor scleral patch was placed over the sclera tunnel to cover the tube. This was sutured to the sclera with $9 / 0$ nylon sutures. The 
Table I Demographic Characteristics of Studied Subjects

\begin{tabular}{|l|l|l|}
\hline & Age (Years) & Sex \\
\hline Sex & Mean \pm Standard Deviation & Male/female (eyes) \\
Shunt & $52.44 \pm 8.91$ & $18 / 14$ \\
Trabeculectomy & $49.69 \pm 6.72$ & $18 / 14$ \\
\hline
\end{tabular}

conjunctiva was reapposed using a $6 / 0$ vicryl suture. The trabeculectomy and shunt surgeries were performed by Fakhraie ophthalmologist.

Data analysis was done by a descriptive statistical method. Correlation between variables was carried out using the Spearman rank correlation equation for nonnormal variables.

A comparison of numeric variables was performed using the Wilcoxon signed-rank test. An analysis of the variables between the two study groups was done via the Mann-Whitney test. All P-values $<0.05$ were considered statistically significant. Data were statistically described in terms of range, mean $\pm \mathrm{SD}$. Changed axis of astigmatism was explained by percent WTR and ATR astigmatism. Correlation between IOP and refractive error was calculated by the Pearson test.

All analyses were performed by SPSS version 16 .

\section{Ethical Issues}

The Ethics Committee of Shahid Beheshti University of Medical Sciences approved the study protocol, which was conducted in accordance with the tenets of the Helsinki Declaration. All participants signed a written informed consent (ethics code: IR SBMU OPT-262).

\section{Results}

In this comparative study, two groups of 16 patients with average age $52.4 \pm 8.9$ and $49.67 \pm 6.7$ were, respectively, studied for trabeculectomy and shunt surgeries. Table 1 shows the demographic characters of both groups. Average intraocular pressure (IOP) before and 1 week, 1 month, and 3 months after trabeculectomy was $31.31 \pm 7.6,9.8$ $\pm 2.8, \quad 10.5 \pm 1.29,10.9 \pm 1.26 \mathrm{mmHg}(P<0.05)$, and in shunt, it was $36.06 \pm 10.32,13.5 \pm 3.3,11.68 \pm 2.21,11.18$ $\pm 1.27 \mathrm{mmHg}(P<0.05)$. Visual acuity comparison before and 1 week, 1 month, and 3 months after trabeculectomy conducted by the Freidman test was significant $(P<0.05)$. The Wilcoxon test showed that visual acuity was lower tree time after surgery than before. After 1 week a gradual increase can be observed in Table 2. In shunt surgery, the result of the Freidman test was significant and visual acuity decreased after surgery, but during 3 months visual acuity was like that before surgery (Table 1); the MannWhitney test revealed that a decrease in visual acuity in trabeculectomy was more than in shunt surgery $(P<0.05)$.

The comparison of the mean of astigmatism amount before trabeculectomy and 1 week, 1 month, and 3 months after trabeculectomy by the Freidman test was significant $(P<0.05)$ and the result of the Wilcoxon test showed that after this surgery the amount of astigmatism was more than before. After 1 month astigmatism decreased, and after 3 months, it was near to that before trabeculectomy. Table 3 compares the mean astigmatism before and 1 month and 3 months after the shunt surgery by the Freidman test and it was not significant $(P>0.05)$. Table 3 in the Mann-Whitney test compares astigmatism among shunt and trabeculectomy and it can be seen that change and access of astigmatism after trabeculectomy was more than the shunt surgery $(P<0.05)$.

The change of axis was reported as a percentage. One week after trabeculectomy, $100 \%$ of the patients were showing with-the-rule astigmatism but 1 month and 3 months after, the percentages of with-the-rule astigmatism were, respectively, $37.5 \%$ and $31.75 \%$. This result shows that the axis tends to against-the-rule astigmatism. But changes in the axis before and after shunt surgery were the same and the percentages of with-the-rule astigmatism 1 week, 1 month, and 3 months after surgery were $50 \%, 50 \%$, and $56.25 \%$.

Table 2 Mean (SD) Visual Acuity Before and I Week, I Month, and 3 Months After Shunt and Trabeculectomy Surgeries

\begin{tabular}{|c|c|c|c|c|}
\hline & Before Surgery & I Week After Surgery & I Month After Surgery & 3 Months After Surgery \\
\hline & Mean \pm SD & Mean \pm SD & Mean \pm SD & Mean \pm SD \\
\hline Trabeculectomy & $1.22 \pm 2.34$ & $\begin{array}{l}0.88 \pm 2.45 \\
P<0.00 I\end{array}$ & $\begin{array}{l}I .03 \pm 2.4 \mathrm{I} \\
P<0.00 \mathrm{I}\end{array}$ & $\begin{array}{l}1.15 \pm 2.38 \\
P<0.001\end{array}$ \\
\hline Shunt & $0.76 \pm 0.16$ & $\begin{array}{l}0.58 \pm 0.24 \\
P<0.00 I\end{array}$ & $\begin{array}{l}0.68 \pm 0.20 \\
P<0.00 I\end{array}$ & $\begin{array}{l}0.75 \pm 0.17 \\
P=0.120\end{array}$ \\
\hline
\end{tabular}


Table 3 Mean (SD) of Astigmatism Before and I Week, I Month, and 3 Months After Shunt and Trabeculectomy Surgeries

\begin{tabular}{|l|l|l|l|l|}
\hline & Before Surgery & I Week After Surgery & I Month After Surgery & 3 Months After Surgery \\
\cline { 2 - 5 } & Mean \pm SD & Mean \pm SD & Mean \pm SD & Mean \pm SD \\
\hline Trabeculectomy & $-1.00 \pm 0.59 D^{*}$ & $\begin{array}{l}-6.00 \pm 1.9 \mathrm{D} \\
P<0.001\end{array}$ & $\begin{array}{l}-2.21 \pm 1.18 \mathrm{D} \\
P=0.003\end{array}$ & $\begin{array}{l}-1.37 \pm 0.62 \mathrm{D} \\
P=0.059\end{array}$ \\
\hline Shunt & & $-1.56 \pm 1.62 \mathrm{D}$ & $-1.51 \pm 1.46 \mathrm{D}$ & $-1.07 \pm 0.85 \mathrm{D}$ \\
& $-0.89 \pm 0.46 \mathrm{D}$ & $P>0.05$ & 0.05 & $P>0.05$ \\
\hline
\end{tabular}

Note: *Diopter unit.

Table 4 Mean (SD) Spherical Equivalent Before, I Week, I Month, and 3 Months After Shunt and Trabeculectomy Surgeries

\begin{tabular}{|l|l|l|l|l|}
\hline & Before Surgery & I Week After Surgery & I Month After Surgery & 3 Months After Surgery \\
\cline { 2 - 5 } & Mean \pm SD & Mean \pm SD & Mean \pm SD & Mean \pm SD \\
\hline Trabeculectomy & $-0.03 \pm 1.49 D^{*}$ & $\begin{array}{l}1.43 \pm 1.94 \mathrm{D} \\
P<0.001\end{array}$ & $\begin{array}{l}0.27 \pm 1.74 \mathrm{D} \\
P=0.34\end{array}$ & $\begin{array}{l}0.04 \pm 1.46 \mathrm{D} \\
P>0.05\end{array}$ \\
\hline Shunt & & $\begin{array}{l}1.03 \pm 2.03 \mathrm{D} \\
P>0.05\end{array}$ & $\begin{array}{l}0.70 \pm 1.64 \mathrm{D} \\
P>0.05\end{array}$ & $\begin{array}{l}0.62 \pm 1.54 \mathrm{D} \\
P>0.05\end{array}$ \\
\hline
\end{tabular}

Note: *Diopter unit.

The mean change of spherical equivalent in trabeculectomy was significant by the Freidman test $(P<0.05)$ and comparison by the Wilcoxon test showed that hyperopia increases after the operation for a month $(P<0.05)$ and during 3 months hyperopia decreases, so the differences in hyperopia before and 1 month and 3 months after surgery were not significant $(P>0.05)$ (Table 4). Results by the Wilcoxon test for shunt operation just showed hyperopia 1 and 3 months after surgery. Another time change in spherical equivalent was not significant $(P>0.05$ ) (Table 4).

Comparison by the Mann-Whitney test showed that change spherical equivalent (shift hyperopia) in trabeculectomy was more than the shunt $(P<0.05)$.

The $P$-value correlation between IOP and refractive error before surgery, 1 week, 1 month, and 3 months after surgery were $0.233,0.259,0.162$, and 0.772 , respectively. So there is no correlation between the two variables.

\section{Discussion}

Trabeculectomy is very effective for controlling the IOP in the long term but results of this study reveal that trabeculectomy produces more changes in corneal curvature than the shunt surgery, and during 1 month of with-the-rule astigmatism, it is more than against-the-rule in trabeculectomy; however, after shunt operation, the percentages of with-the-rule and against-the-rule astigmatism were the same.
Equivalent sphere (hyperopia) during 2 months after trabeculectomy was more than before but in shunt there was not much difference before and after operations and some hyperopia was observed. So these changes in the refraction of eyes decrease the visual acuity of patients after trabeculectomy. As shown in this study, visual acuity decreases in trabeculectomy and for 3 months after surgery. Most studies revealed induced with-the-rule astigmatism with a mean of $0.81 \pm 1.083$ months after trabeculectomy. The first study by Hugkulstone demonstrated a reduction in vertical corneal radius of curvature for 7 weeks after trabeculectomy. ${ }^{6}$ Rosen et al found a mean of $1.24 \mathrm{D}$ withthe-rule in astigmatism 3 months after trabeculectomy. ${ }^{15}$ Claridge et $\mathrm{al}^{16}$ observed astigmatism 1 and 3 months after trabeculectomy, with a mean of $1 \mathrm{D}$. Cunliffe et $\mathrm{al}^{12}$ investigated the effects of trabeculectomy on 16 eyes for 2 months. Results revealed that WTR astigmatism was more sudden after surgery and it decreased over 10 months.

According to the conducted studies, there are many reasons for induced astigmatism after trabeculectomy. Vernon et al evaluated the effect of mini trabeculectomy ( $2 \times 2 \mathrm{~mm}$ scleral flap) on corneal astigmatism and reported a lower amount of induced astigmatism. However, using antimetabolites at the time of surgery, with-the-rule astigmatism stays longer on the cornea postoperatively. ${ }^{17}$ Cunliffe et $\mathrm{al}^{12}$ proposed that internal sclerostomy retracts the corneal edge and subsequently reduces vertical corneal 
radius. Rosen et $\mathrm{al}^{15}$ stated that cauterization at the time of surgery is the main factor of changes in corneal curvature. Mokhtari investigated changes in corneal topography before and after shunt surgery which revealed that shunt operation has no significant effect on corneal curvature $(P>0.05){ }^{14}$

One reason for induced hyperopia after trabeculectomy was the reduction of $0.1-0.9 \mathrm{~mm}$ in axial length; this would stabilize almost 3 months after this surgery., ${ }^{4,6,12}$

Cash well and Martin evaluated 62 patients who underwent trabeculectomy, and the mean of axial length after surgery was reduced to $0.423 \pm 0.61 \mathrm{~mm}$ after 22.5 months. ${ }^{18}$ Kook et al ${ }^{19}$ reported a reduction in axial length lower than other studies after trabeculectomy with mitomycin-C (MMC). Francis et $\mathrm{al}^{20}$ measured axial length after trabeculectomy by IOL Master that was reduced to $0.16 \pm 0.03 \mathrm{~mm}$ in the group who developed hypotony. Axial length was reduced 3 times greater in the patients without hypotonic.

No study has been conducted comparing the axial length before and after shunt surgery, but the results of this study and another ${ }^{14}$ show that shunt influences the refraction of eyes, but visual function induced by changes in corneal curvature and the equivalent sphere following trabeculectomy are annoying both for the patients and the surgeons. This is because there are complaints about the reduction of vision after successful glaucoma surgery, particularly when changes are marked and continue. In our study, there is no correlation between IOP and spherical equivalent before surgery and 1 week, 1 month, and 3 months after surgery $(P>0.05)$.

But $P$-value between IOP and astigmatism 1 week after surgery was 0.016 , which showed a correlation between two variables.

Thus, according to the results of this study and other studies, shunt surgery is safe, and regarding refractive errors, it might be a better method. But selection of that type of surgery that depends on the patient's eye condition and doctor's discretion.

The limitations of this study are the small population and small period of followup; therefore, these bias the results of this study. Because the scleral flap was not the same in all patients, the thickness of the removed flap should have been mentioned and its relation to the refractive error measured postoperatively.

We suggest that further studies be conducted for differential diagnoses that induce hyperopia due to a decrease in axial length or anterior chamber. It is suggested that in subsequent studies, axial length be measured after shunt and trabeculectomy.

Correct refractive error is a better prescript after 3 months in trabeculectomy and shunt surgery because at this time the refractive state of the eye is stable.

\section{Acknowledgment}

This project was supported by the Shahid Beheshti University of Medical Science.

\section{Disclosure}

The authors report no conflicts of interest in this work.

\section{References}

1. Ophthalmology. CGAAo. Glaucoma. España: Elsevier S; 2011-2012.

2. WHO. Glaucoma Is Second Leading Cause of Blindness Globally; 2017.

3. Kingman S. Glaucoma is second leading cause of blindness globally. Bull World Health Organ. 2004;82:887-888. doi:10.1590/S004296862004001100019

4. Benjamin WJ. Borish's Clinical Refraction. Elsevier Health Sciences; 2006.

5. Cairns JE. Trabeculectomy. Preliminary report of a new method. Am J Ophthalmol. 1968;66:673-679. doi:10.1016/0002-9394(68)91288-9

6. Hugkulstone CE. Changes in keratometry following trabeculectomy. Br J Ophthalmol. 1991;75:217-218. doi:10.1136/bjo.75.4.217

7. Molteno AC. New implant for drainage in glaucoma. Animal trial. $\mathrm{Br}$ J Ophthalmol. 1969;53:161-168. doi:10.1136/bjo.53.3.161

8. Eisenberg DL, Koo EY, Hafner G, et al. In vitro flow properties of glaucoma implant devices. Ophthalmic Surg Lasers. 1999;30:662-667.

9. Francis BA, Cortes A, Chen J, et al. Characteristics of glaucoma drainage implants during dynamic and steady-state flow conditions. Ophthalmology. 1998;105:1708-1714. doi:10.1016/S0161-6420(98) 99042-X

10. Stay MS, Pan T, Brown JD, et al. Thin-film coupled fluid-solid analysis of flow through the Ahmed glaucoma drainage device. $J$ Biomech Eng. 2005;127:776-781.

11. Kumari R, Saha BC, Puri LR. Keratometric astigmatism evaluation after trabeculectomy. Nepal J Ophthalmol. 2013;5:215-219. doi:10.3126/nepjoph.v5i2.8731

12. Cunliffe IA, Dapling RB, West J, et al. A prospective study examining the changes in factors that affect visual acuity following trabeculectomy. Eye (London, England). 1992;6(Pt 6):618-622. doi:10.1038/ eye. 1992.133

13. Popa-Cherecheanu A, Iancu RC, Schmetterer L, et al. Intraocular pressure, axial length, and refractive changes after phacoemulsification and trabeculectomy for open-angle glaucoma. $J$ Ophthalmol. 2017;2017:1203269. doi:10.1155/2017/1203269

14. Mokhtari S. Changes in Corneal Topography Before \&after Shunt Surgery in Cronic Glaucoma. Shahid Beheshti University of Medical Sciences; 2014.

15. Rosen WJ, Mannis MJ, Brandt JD. The effect of trabeculectomy on corneal topography. Ophthalmic Surg. 1992;23:395-398.

16. Claridge KG, Galbraith JK, Karmel V, et al. The effect of trabeculectomy on refraction, keratometry and corneal topography. Eye (London, England). 1995;9(Pt 3):292-298. doi:10.1038/eye.1995.57

17. Vernon SA, Zambarakji HJ, Potgieter F, et al. Topographic and keratometric astigmatism up to 1 year following small flap trabeculectomy (microtrabeculectomy). Br J Ophthalmol. 1999;83:779-782. doi:10.1136/bjo.83.7.779 
18. Martinez JA, Brown RH, Lynch MG, et al. Risk of postoperative visual loss in advanced glaucoma. Am J Ophthalmol. 1993;115:332337. doi:10.1016/S0002-9394(14)73584-6

19. Kook MS, Kim HB, Lee SU. Short-term effect of mitomycin-C augmented trabeculectomy on axial length and corneal astigmatism. J Cataract Refract Surg. 2001;27:518-523. doi:10.1016/S0886-3350(00)00646-5
20. Francis BA, Hong B, Winarko J, et al. Vision loss and recovery after trabeculectomy: risk and associated risk factors. Arch Ophthalmol. 2011;129:1011-1017. doi:10.1001/archophthalmol. 2011.182

\section{Clinical Optometry}

\section{Publish your work in this journal}

Clinical Optometry is an international, peer-reviewed, open access journal publishing original research, basic science, clinical and epidemiological studies, reviews and evaluations on clinical optometry. All aspects of patient care are addressed within the journal as well as the practice of optometry including economic and business analyses. Basic and clinical research papers are published that cover

Submit your manuscript here: https://www.dovepress.com/clinical-optometry-journa

\section{Dovepress}

all aspects of optics, refraction and its application to the theory and practice of optometry. The manuscript management system is completely online and includes a very quick and fair peer-review system, which is all easy to use. Visit http://www.dovepress.com/ testimonials.php to read real quotes from published authors. 